\title{
Deformable Cell Model and its Application to Growth of Plant Meristem
}

\author{
N. Bessonov ${ }^{1}$, V. Mironova ${ }^{2}$, V. Volpert ${ }^{3,4} *$ \\ ${ }^{1}$ Institute of Mechanical Engineering Problems, 199178 Saint Petersburg, Russia \\ ${ }^{2}$ Institute of cytology and genetics SB RAS, Novosibirsk, Russia \\ ${ }^{3}$ Institut Camille Jordan, UMR 5208 CNRS, University Lyon 1, 69622 Villeurbanne, France \\ ${ }^{4}$ Department of Mathematics, Mechanics and Computer Science \\ Southern Federal University, Rostov-on-Don, Russia
}

\begin{abstract}
Deformable cell model is developed to study pattern formation and to simulate plant tissue growth. Each cell represents a polygon with a number of vertices connected by springs. Some cells in the tissue can grow and divide, other cells are differentiated and do not grow or divide but remain deformable. The model is used to investigate formation of self-similar structures which reproduce the same cell organization during their growth. In numerical experiments we observed that self-similar solutions can exist for a rather precise choice of plant structure and mechanical properties of cell walls. We test the model for simulation of apical meristems functioning which represent self-similar cell structures in plants. At the next stage of modelling, auxin distribution is introduced by means of diffusion and polar transport mechanisms. The existence of steady auxin distribution in a growing root is investigated. Single as well as multiple auxin maxima have been observed in model solutions.
\end{abstract}

Keywords and phrases: deformable cell model, self-similar solution, root apical meristem, auxin transport

Mathematics Subject Classification: 92C80

\section{Introduction}

Meristems are the stem cell niches in the plant. All aboveground plant organs are derived from the shoot apical meristem (SAM). Underground part is generated by the root apical meristem (RAM). In higher plants the meristems are established in the embryo, and are organized into distinct layers and domains. This structure maintains although stem cells continuously leave the meristem and are replaced by their descendants.

In the SAM stem cells are located in the so called central zone at the top of the meristem [28]. They are arranged into three layers: epidermal layer (L1), subepidermal layer (L2) and cortex (L3). Stem cells from L1 and L2 divide only anticlinally giving rise two continuous layers of tunica. The stem cells from L3 divide in random planes providing for volume growth of the shoot.

${ }^{*}$ Corresponding author. E-mail: volpert@math.univ-lyon1.fr 
In the RAM stem cells are located around a quiescent center (QC), which has low mitotic activity [13]. Below the QC root cap initials generate the root cap, which covers and protects the RAM. As root grows, the outer layer of the root cap is continuously sloughed away and is replaced by the descendants of the root cap initials. Above the QC vascular initials provides for development of vascular cylinder. The initials located sideways to the QC give rise to epidermis, endodermis and cortex. The RAM of the model plant Arabidopsis thaliana has a small size and consists of the four-cell QC encircled by one layer of initials [10].

Maintenance and functioning of SAM and RAM is guided by hormone auxin. Auxin mainly is synthesized in the shoots and is transported toward the root tips by means of diffusion and active transport. By the auxin responsive reporters DR5 and DII it has been revealed that auxin distribution has a maximum in the stem cells of both SAM and RAM of Arabidopsis thaliana [8], [23]. The main contributors to auxin patterning in the SAM and RAM are the auxin carriers of PIN family [6], [12]. PIN proteins are polarly localized to cell membranes directing auxin transport in plant tissue. For example, PIN1 protein is expressed in the vascular cylinder of the RAM with rootward polarization [6], [27]. It has been shown that auxin regulates PIN1 expression both positively and negatively [23], [27]. The mechanism of auxin patterning in the root apical meristem which accounts for this dual regulation was called the reflected flow mechanism [20]. It participates in various physiological processes in the root [21].

There are numerous approaches to plant growth modelling (see, e.g., a recent review [18]). Essential part of these studies is devoted to auxin transport mechanisms [7], [17]. Auxin transport as well as its feedbacks to an organ development were investigated for the SAM [2], [15], [24]; in the RAM [1], [14], [21]; in leaves [2], [5]. Two-dimensional models of auxin transport are developed in [11], [16]. In the most part of the existing models auxin transport is investigated in a fixed ensemble of cells. However, several aspects of cell dynamics in the meristem have been investigated for phyllotaxis [15], [19], [24] and for root growth [20], [25], [26]. Branching patterns in plants depending on auxin and cytokinin interaction is studied in [3], [4].

In this work we develop a deformable cell model for simulation of plant tissue growth. Cells can grow, change their shape due to the mechanical interaction with the surrounding cells and they can divide. There are different cell types. Stem cells (or initials) grow and divide usually producing one cell similar to the mother cell and one differentiated cell. An important parameter of dividing cells is the direction of cell division. We will show that the properties of growing cell structures crucially depend on it.

One of the basic features of plants is self-similarity of its modules. We formalize the notion of selfsimilarity of cell structures. A growing cell structure $S(t)$ is self-similar if for any time $t$ it can be obtained from $S(t-\tau)$ by translation in space. Here $\tau$ is some time delay. A more precise definition used in this work will be given below.

We will apply the deformable cell model to search self-similar growing structures. From numerical simulations it appears that they exist under specific and rather restrictive conditions on cell organization, division angles and mechanical properties. At the next step of this study, we introduce in the model some properties specific for root and shoot meristems and we will show the existence of the corresponding self-similar growing structures. We note that the model developed in this work is a simplified model to study the mechanisms of pattern formation. It is based on cell deformation properties but it is not aimed to predict stresses and displacement in real plants.

In the last part of this work we will investigate auxin transport along self-similar growing structures simulating root growth. We will test the mechanism of reflected flow [20] which takes into account the feedbacks from auxin to its own transport. In the stationary model solution we observe formation of single as well as multiple auxin maxima along the root. 


\section{Growth of cellular structures}

\subsection{Mechanical model}

We will consider structures formed by deformable cells (Figure 1, a). Deformable cells are used in modelling of plant growth in [19]. We begin the description of the model with mechanical forces acting in cellular structures. An individual cell at the equilibrium represents a regular polygon with $n$ vertices. Initially it has an area $S_{0}$, the length of the sides $l_{0}$ and the angle between any neighboring sides $\alpha_{0}$. These parameters can be different for different cells. We place a particle of mass $m$ at each vertex of the polygon.

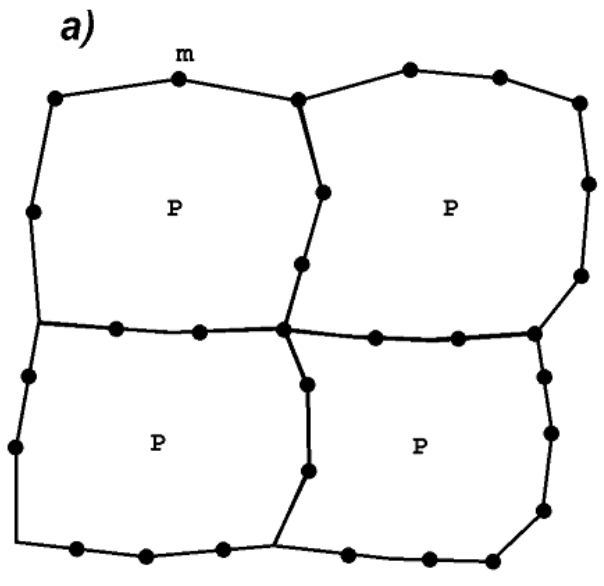

b)

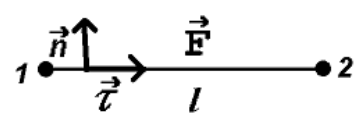

c)

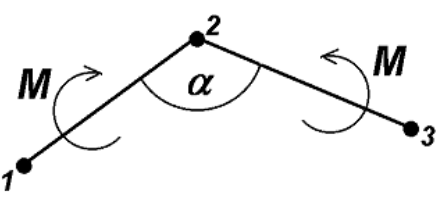

d)

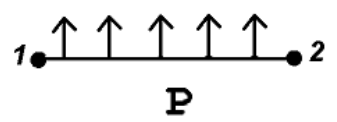

FiguRE 1. Schematic representation of the model: a structure which consists of four deformable cells (a), stretching force acts between two neighboring vertices along the line connecting them (b), bending force acts on neighboring sides is the angle between them differs from the equilibrium angle $\alpha_{0}$ (c), pressure acts on all sides in the direction perpendicular to them (d).

If the polygon changes its shape, then there are three forces which tend to return it to its equilibrium configuration. They depend on the change of side length, angles and volume. Similar to other deformable cell models, we define them as follows. If the length $l$ of a side changes, then there is a force acting on each of the two vertices located at this side:

$$
\vec{f}_{1}=\left(k_{1}\left(\frac{l}{l_{0}}-1\right)+\mu_{1} \frac{d l}{d t}\right) \vec{\tau}, \quad \vec{f}_{2}=-\vec{f}_{1}
$$

(Figure 1, b). Here $\vec{\tau}$ is the unit vector along the side. If the angle $\alpha$ between two sides changes (Figure $1, \mathrm{c})$, then there is a momentum

$$
M=k_{2}\left(1-\frac{\alpha}{\alpha_{0}}\right)-\mu_{2} \frac{d \alpha}{d t}
$$

which creates a pair of force applied to the vertices and acting on each side in the direction perpendicular to it. Finally, if the cell area $S$ changes, then there is a force (pressure) 


$$
P=k_{3}\left(1-\frac{S}{S_{0}}\right)-\mu_{3} \frac{d S}{d t}
$$

applied to each side and acting in the direction perpendicular to it (Figure 1, d). Let us note that the first terms in (2.1)-(2.3) describe elastic force and the second term viscous dissipation. They correspond to visco-elastic properties of biological cells. The typical values of parameters which we take in the simulations below are $k_{1}=10, k_{2}=10, k_{3}=100, \mu_{i}=1 \div 10, i=1,2,3$ (dimensionless values).

The total force $\vec{F}_{i}$ acting on the $i$-th vertex is a sum of these three forces. The position $\vec{r}_{i}$ of this vertex is determined by the equation

$$
m_{i} \frac{d \vec{r}_{i}}{d t}=\vec{F}_{i}
$$

We introduce two additional features in the model:

1. If the length of a side becomes twice larger than its initial length $l_{0}$, then an additional vertex is introduced in the middle of the side. In the moment of side division, the stretching force is preserved. Hence the number of vertices depends on cell deformation. This allows us to better describe cell shape in the case of large deformations.

2. If the length $l$ of a side becomes sufficiently large, that is $l_{0} / l \leq \gamma$, with some $\gamma, 0<\gamma<1$, then the initial length $l_{0}$ increases irreversibly in such a way that it satisfies the equality $l_{0}=\gamma l$. In this case, if the stretching force is removed, then the spring will not return to its initial length but to some greater length. This corresponds to irreversible deformation of cell wall when it growth.

The equilibrium area $S_{0}$ of growing cells changes in time with the rate:

$$
\frac{d S_{0}}{d t}=\lambda e^{-a t}
$$

where $\lambda$ and $a$ are positive constants. Their values can be different for different cells. If a cell does not grow, we set $\lambda=0$. We have linear growth if $a=0$.

When $S_{0}$ increases twice, the cell divides. The equilibrium area of the daughter cell returns to the twice less value. When studying root growth, we will also introduce cell growth without division.

2.1.0.1. Cell division. Important parameter is the direction of cell division. It is specified for each dividing cell in the following way. Cell is divided by a straight line. Suppose that we want this line to form a given angle $\alpha$ from the horizontal direction counterclockwise. Let us recall that cell is a polygon. We consider all diagonals of this polygon, which divide it into two polygons with approximately equal areas. Among all such diagonals, we chose that one which is the closest to the given direction $\alpha$. Hence the angle of division is not exact but approximate. Increasing the number of vertices in the polygon, we improve the approximation.

2.1.0.2. Software. All numerical simulations in this work are carried out with the original software developed by the authors. In order to develop a simulator for the described model, $\mathrm{C}++$ programming language was used. The integrated development environment was MS Visual Studio 2008, accompanied with Microsoft Foundation Classes (MFC) and OpenGL for the development of the graphical user interface, OpenMP for parallelization. Detailed description of the software will be presented elsewhere.

\subsection{Growth of self-similar structures}

2.2.0.3. Formulation of the problem about self-similar growing structures. Plant meristems represent self-similar cell structures which reproduce the same cell organization during their growth. They are composed of dividing cells (stem cells or initials) and differentiated cells which do not divide any more. This self-similarity implies certain restrictions on location of dividing and differentiated cells and on their mechanical properties. Let us consider a schematic example shown in Figure 2. The initial structure at the left consists of a square grid with one green cell at the center. This is the only cell which grows and 

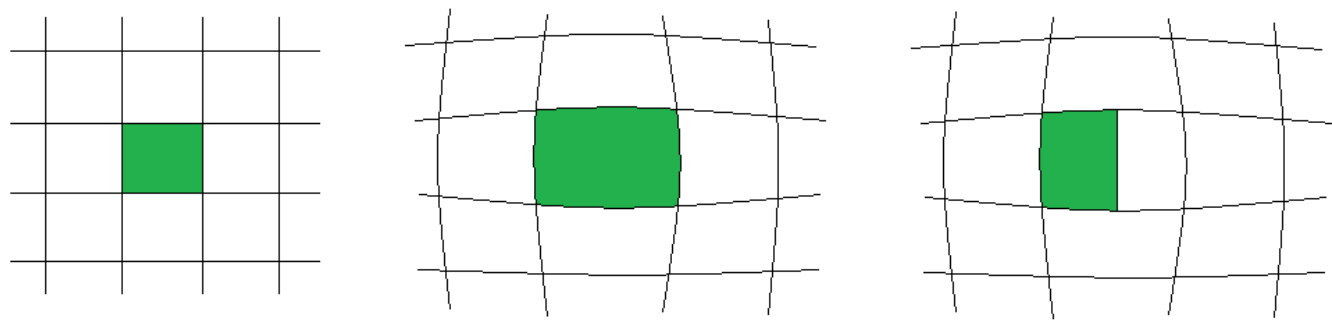

Figure 2. Schematic representation of cell growth and division. The green cell in the center grows (middle) and divides (right) into one green cell and one white cell. The structures at the left and at the right are topologically different: at the left the green cell is surrounded by 8 white cells while in the right by 6 cells.

divides. After growing twice (middle) it divides into one green cell and one white cell. In the example presented in Figure 2 the structure at the right figure is topologically different from the structure at the left. Indeed, the green cell is surrounded by eight white cells at the left and by six cells at the right. We are interested here by the question whether we can get the same structure after growth and division as before. If it is possible, we will call them self-similar growing structures.
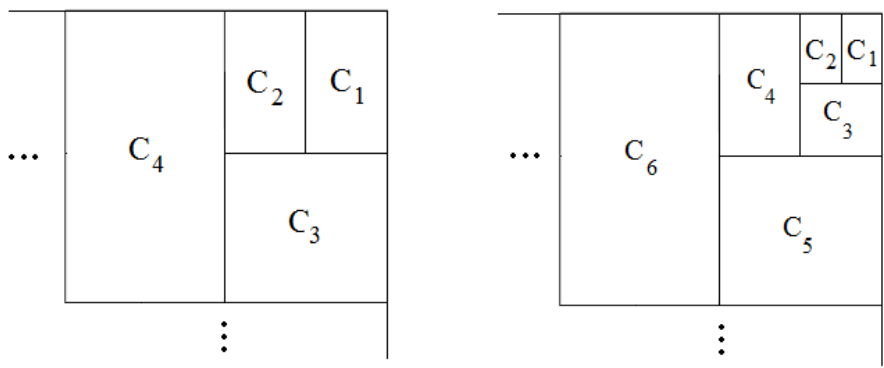

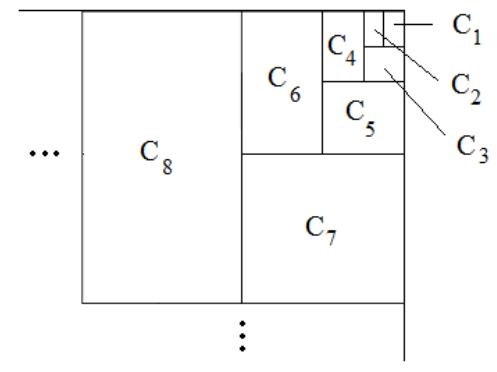

FiguRE 3. An example of 2-topologically equivalent structure in a quarter plane. Only a finite number of cells is shown. The cell $C_{1}$ in the left structure divides twice giving the structure in the middle. After the next two divisions, we obtain the structure at the right. The structures are topologically equivalent.

The formal definition of self-similar growing structures is as follows. Two cell structures are called equivalent if they can be superposed by translation and rotation in such a way that all cells of one structure coincide by form and type with cells of the other one. A cell structure is called self-similar growing structure if after a finite number of growth/division processes it becomes equivalent to the original structure.

Since there are dividing cells, then their number grows unless it is infinite. Hence exact self-similar growing structure can be obtained only for an infinite number of cells. However we can also consider quasi-self-similar structures which we will understand as follows. We consider a finite number of cells which contain all green cells among them. If these group of cells preserve their structure after division, then we call this structure quasi-self-similar. In what follows we will consider only such structures and will call them, for brevity, self-similar. 
There is a simple example of self-similar growing structures. It is a rectangle where dividing cells are located at one of its sides. After each division, a new layer of differentiated cells is added inside and dividing cells remain at the outer layer.

We will search other self-similar structures numerically. Though these solutions do not take into account all features of root or shoot meristem organization, they reflect their main property, self-similarity. Taking into account that numerical solutions are approximate, we cannot expect the exact self-similarity in the sense of the definition given above. Therefore we will introduce a more general definition of topological self-similarity. Consider a cell structure composed of a finite or infinite number of cells. Each of the cells represents a polygon. Let us introduce a numbering of cells and denote them by $C_{1}, C_{2}, \ldots$ For each cell $C_{k}$ we determine all cells, which have a common boundary with the cell $C_{k}$, and all cells, which have a common vertex with the cell $C_{k}$. Two cell structures are called topologically equivalent if there exists such numbering that for each cell $C_{k}$ of each structure the sets of cells with common boundaries and with common vertices are the same.

Consider now a structure where some cells can divide. A growing structure is called n-topologically self-similar, if after each $n$ division processes the new structure is topologically equivalent to the old structure.

The left and the right figures in Figure 2 are not topologically equivalent. An example of topologically equivalent self-similar structure is shown in Figure 3. The original structure is at the left. Though only four cells are shown, it can be continued in an obvious way to a cell structure with an infinite number of cells which fill the quarter plane. The cell $C_{1}$ divides. First by a horizontal line, and then the upper of the two new cells by a vertical line. We obtain the structure shown in Figure 3, middle. This new cell structure is topologically equivalent to the previous one. The structure after the next two divisions is shown in the right figure.

Let us consider the cell structures in Figure 3. We put the origin in the upper right corner and introduce the polar coordinates $(r, \phi), r \geq 0, \pi \leq \phi \leq \frac{3}{2} \pi$. Then the change of variables $(r, \phi) \rightarrow(2 r, \phi)$ transforms the second structure in the first one, and the third structure in the second one. Therefore growth and division gives here the exact self-similarity in the sense of the first definition.

This example can generate other self-similar growing structures. In particular, we can extend it by symmetry on the half-plane. Self-similar growing structures in cylindrical domains can be obtained by conformal mappings.

2.2.0.4. Self-similar structure with two layers of dividing cells. We begin with the example shown in Figure 4. Let us describe the initial cell configuration (Figures 4, a). We compose the initial structure from individual deformable cells with the properties described in the Section 2.1. There are three cell types in this model. Green cells or initials, which will grow and divide, yellow cells or differentiated cells do not grow or divide. Both of them are deformable in the sense described in the previous section. Grey cells at the lower row provide a support. They do not grow and keep their square shape.

The growing structure is shown in Figure 4, b and its tip in Figure 4, c. Green cells change their volume linearly in time with a given rate. Any polygon side which belongs to two cells remains their common side in the process of growth. Therefore growth of green cells leads to their deformation and also to deformation of yellow cells.

When green cells increase twice their area, they divide into two cells with approximately equal areas. One of the two cells remains green (initial cell), that will grow and divide, another one becomes yellow. Green cells are always chosen adjacent to the vertical axis of symmetry.

We need to specify directions of division for green cells. The left green cell in the upper row divides into a green cell adjacent to the middle line and a yellow cell to the left from it. The straight interval dividing them is directed at $2 \pi / 3$ with respect to the horizontal direction. This interval becomes a cell wall with a number of vertices placed along it. The green cell in the right column divides in a similar way, symmetric with respect to the axis. The left green cell in the second row divides along the direction $3 \pi / 4$. The angles indicated above are imposed as parameters of the corresponding cells. 
a
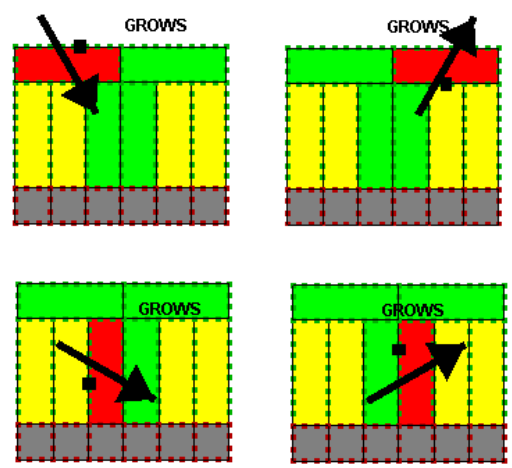

f
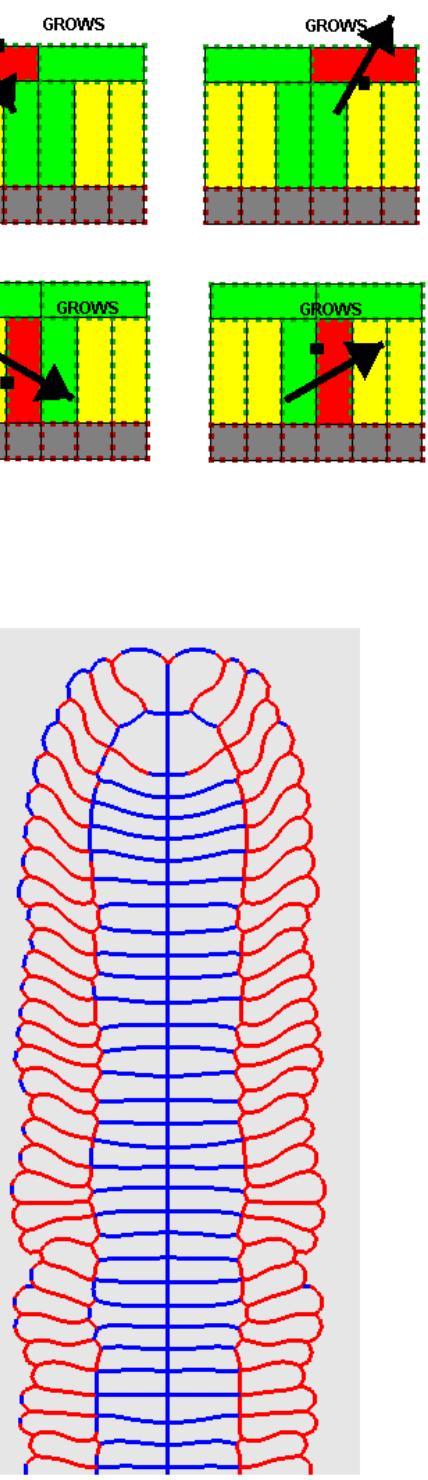

$\mathrm{b}$
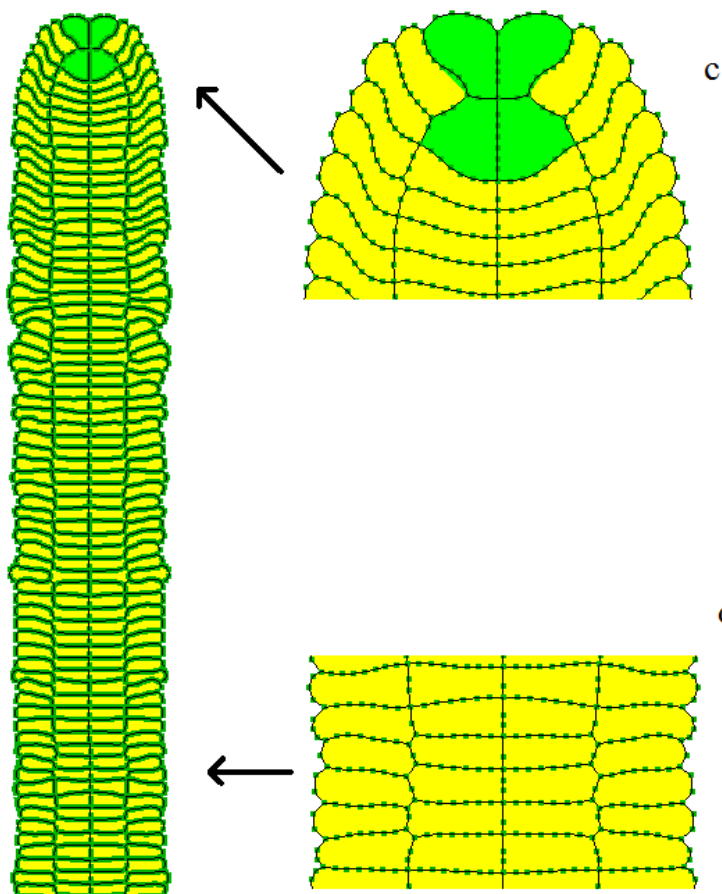

$\mathrm{e}$

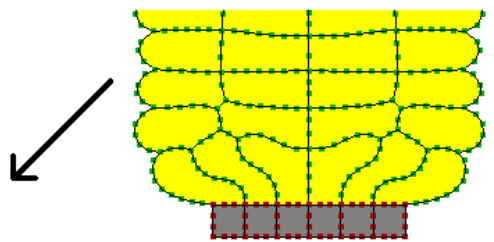

FiguRE 4. Self-similar growth with four cells in each horizontal row and two layers of dividing cells. Initial configuration and directions of division (a), snapshot of the structure (b), zoom on some its parts (c-e), stress distribution (f). The colors in figure (f) indicate compressed (red) or stretched (blue) cell walls. 
The choice of division angles is very important for the existence of self-similar growing structure. If it is done in a wrong way, then the structure will rapidly lose its self-similarity(after one or several divisions). This choice is probably non-unique. It is done in numerical simulations empirically by optimization of the properties of the structure: it should allow unlimited growth which preserves its central symmetry. If the angles are slightly different from those indicated above, then the structure will still exist though the form of the cells can also be slightly different. However, if the values of the angles are changed more essentially, the structure will grow during some time but then it will lose its symmetry due to stress accumulation and then rapidly disintegrate. If the angles strongly differ from the optimal values, then the structure will change its topology after the first division and will not allow self-similarity.
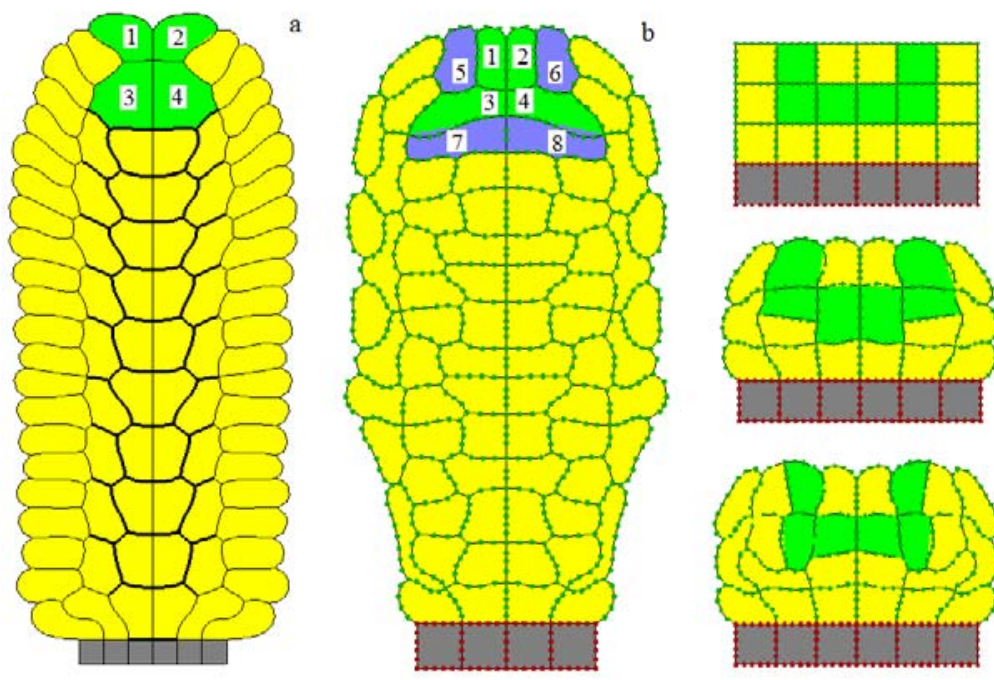

c1

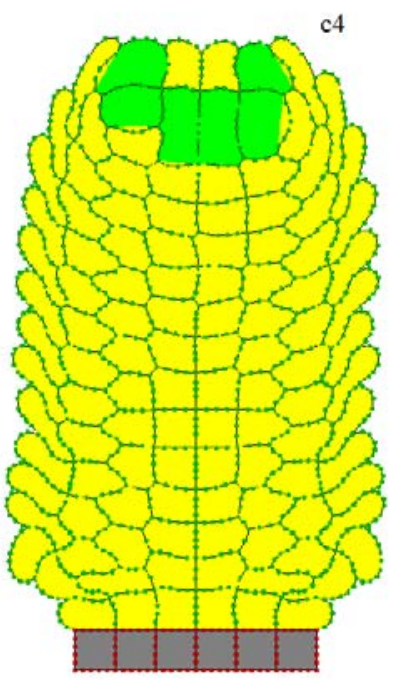

FiguRE 5. a) Self-similar structure with variable directions of cell division. Cells 3 and 4 alternate horizontal and vertical divisions; b) Self-similar structure with one more cell type: green cells divide into green and blue, blue cells divide into two yellow cell (see the explanation in the text); c) Root growth with quiescent center (yellow cells in the center at the top layer). Initial configuration (c1), two consecutive divisions in different directions (c2) and (c3), growing root (c4).

2.2.0.5. Stresses. One of the important questions in growth of self-similar elastic structures is related to distribution of stresses. Even if such structure exists, it can lose its stability because of mechanical stresses, and we will not be able to observe it numerically. For a given geometrical structure stresses depend on the parameters which determine mechanical properties of cell walls and on directions of cell division. All these parameters are chosen in numerical simulations in order to minimize stresses accumulating during plant growth. Examples of stress distribution for self-similar structure with two layers of dividing cells are presented in Figure 4, e. Compressed cell walls are shown in red, stretched walls in blue.

We can remark that cell walls are basically compressed in the exterior cells layers and stretched for the interior cells. The cell walls at the vertical symmetry line can be compressed since they are pushed upwards by growing cells from below. As a result, similar to the elastic rode under load, they can lose their stability and bifurcate into an asymmetric configuration. After that stresses rapidly accumulate. Cell walls becomes strongly deformed and numerical algorithm fails.

2.2.0.6. Other examples of self-similar growing structures. We have presented above one of possible types of self-similar growing structures with two columns and two layers of dividing cells. Directions of 

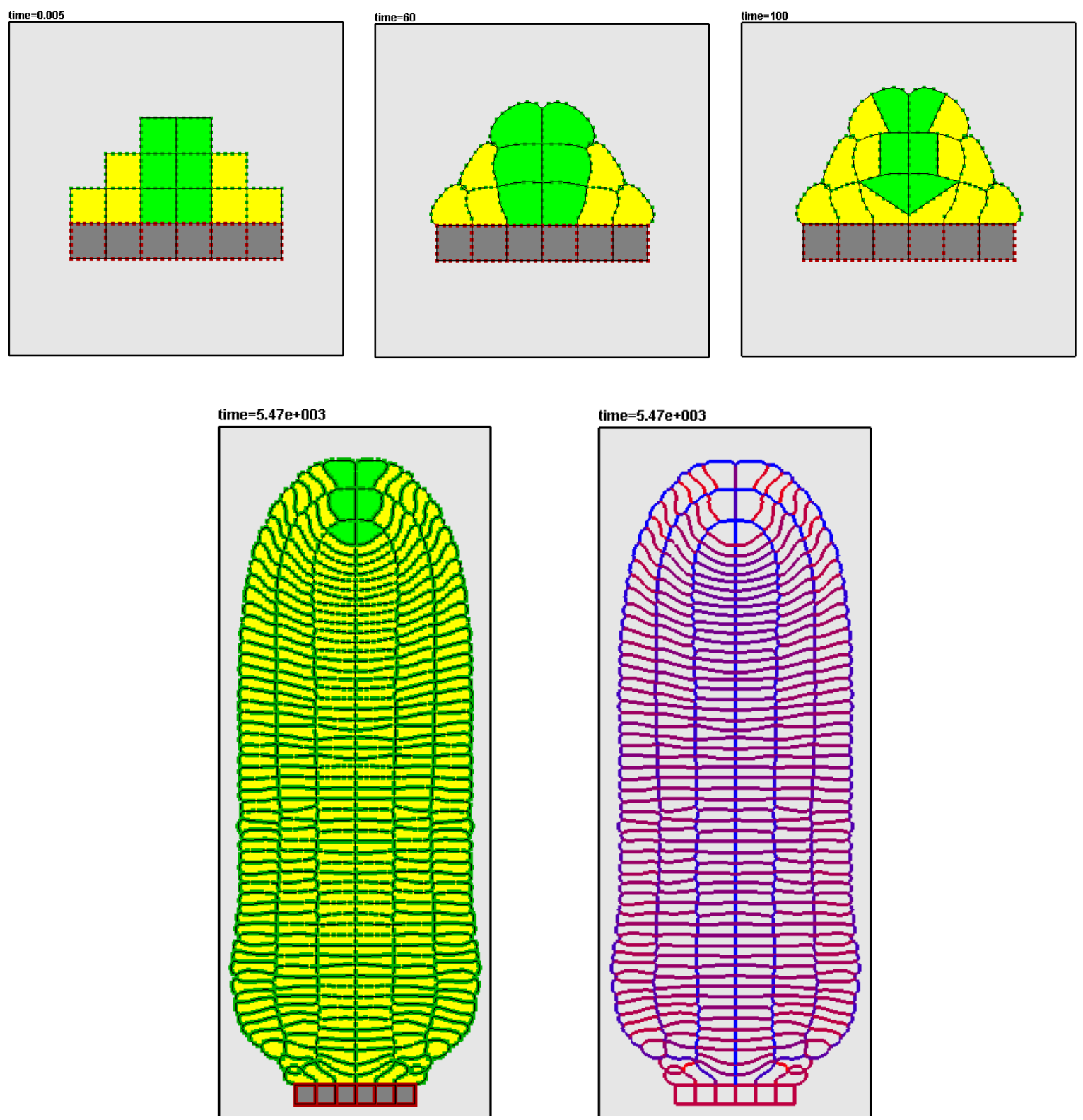

Figure 6. Top: initial condition and the beginning of growth with three layers of dividing cells. Green cells grow and divide, yellow cell do not grow or divide but remain deformable, grey cells are fixed (rigid). Directions of cell division can be seen after the first division at the right figure: the left upper cells divides along $2 \pi / 3$, the left middle cell along $\pi / 2$, the left lower cell along $3 \pi / 4$. Bottom: self-similar structure grown from the cellular layout in Figure 6. Distribution of stresses in the same structure (right). Compressed walls are shown in red, stretched walls in blue. 
cell division are considered as fixed parameters (they can be different for different cells). We will present here some other examples.

In the first one (Figure 5, a) direction of division is not fixed for some of the cells, it changes from one division to another one. Cells 1 and 2 have constant directions of division, respectively $2 \pi / 3$ and $\pi / 3$. Cells 3 and 4 have variable directions of division where horizontal and vertical directions alternate. This structure provides stable growth.

Another example of self-similar growing structures is shown in Figure 5, b. In this case we introduce one more cell type. Green cells divide now into one green cell and one blue cell. Each blue cell divides producing two yellow cells. In Figure 5, b, green cell 1 divides into the same cell and blue cell 5; green cell 3 divides into 3 and 7 . Blue cell 5 divides into two yellow cells to the left of it, blue cell 7 into two yellow cells below it.

Thus, we have two types of differentiated cells, blue and yellow. Though blue cells grow and divide, they do not reproduce other blue cells after division. So they are qualitatively different in comparison with green cells which have this property of self-reproduction specific for stem cells.

Another important difference of blue cells is that we do not fix their direction of division. They divide along the shortest distance which separate them into two equal parts. Let us recall that cells are polygons. So among all diagonals of the polygon corresponding to the blue cell, we choose the shortest one which divides its area in the proportion closest to $1 / 2$. This means that the cell area is preserved after division approximately and not precisely.

The last example is shown in Figures 5, c1-c4. This cell organization is specific for root growth with a quiescent center. We will return to it in the next section.

2.2.0.7. Three layers of dividing cells. Shoot apical meristem (SAM) is generated by stem cells located at the top of the shoot and organized into three layers (L1, L2, L3). To simulate SAM-like self-similar structure formation we used the initial cell configuration with three layers of dividing cells, two cells per layer (Figure 6, top left). Green cells increase their area linearly in time (Figure 6, top middle). When it increases twice, the cell divides (Figure 6, top right).

In real SAM stem cells of different layers divide in different directions: L1 and L2 divide anticlinally, L3 cells divide in variable directions. In the simplified SAM-like structure shown in Figure 6 we introduce the following rules of division. The first division is shown in Figure 6 (right). The left green cell in L1 divides at $2 \pi / 3$ with respect to the horizontal direction; L2 green cell at $\pi / 2$ and the cell below it along $3 \pi / 4$. As a result, the self-similar growing structure shown in Figure 6 is produced. Likely to the real SAM, continuous L1 and L2 layers are generated from activity of upper green cells. The inner green cells produce highly organized two-layers corpus unlike the real SAM whereas L3 stem cells divide in different directions.

Stress distribution is shown in (Figure 6, bottom right). The color code here is different compared with the previous case (Figure 4). The brightness of the color increases with the value of stresses.

\section{Root growth models}

In this section we will use self-similar structures to study root growth. In agreement with the previous section, we will modify the model to describe some specific properties of root apical meristem (RAM). In the RAM, initials are located around slowly dividing quiescent center (QC). Lower initials called root cap initials generate columella, which protect the meristem from mechanical damages. Columella consists of several layers of quite big cells which periodically sloughed off. Upper and side initials to QC divide in different directions providing generation of concentric layers of the root.

We will use the deformable cell model to construct a self-similar growing structure of RAM. We will consider two specific features of growing roots: quiescent center and growth and division of differentiated cells. In order to simplify the model, we do not consider columella here.

3.0.0.8. Quiescent center. The QC maintenance in development is extremely important for root growth. In the deformable cell model we will consider the QC as non dividing cells and will investigate existence of a self-similar solution. As a first approach, we will not take into account cell dynamics of the root cap 


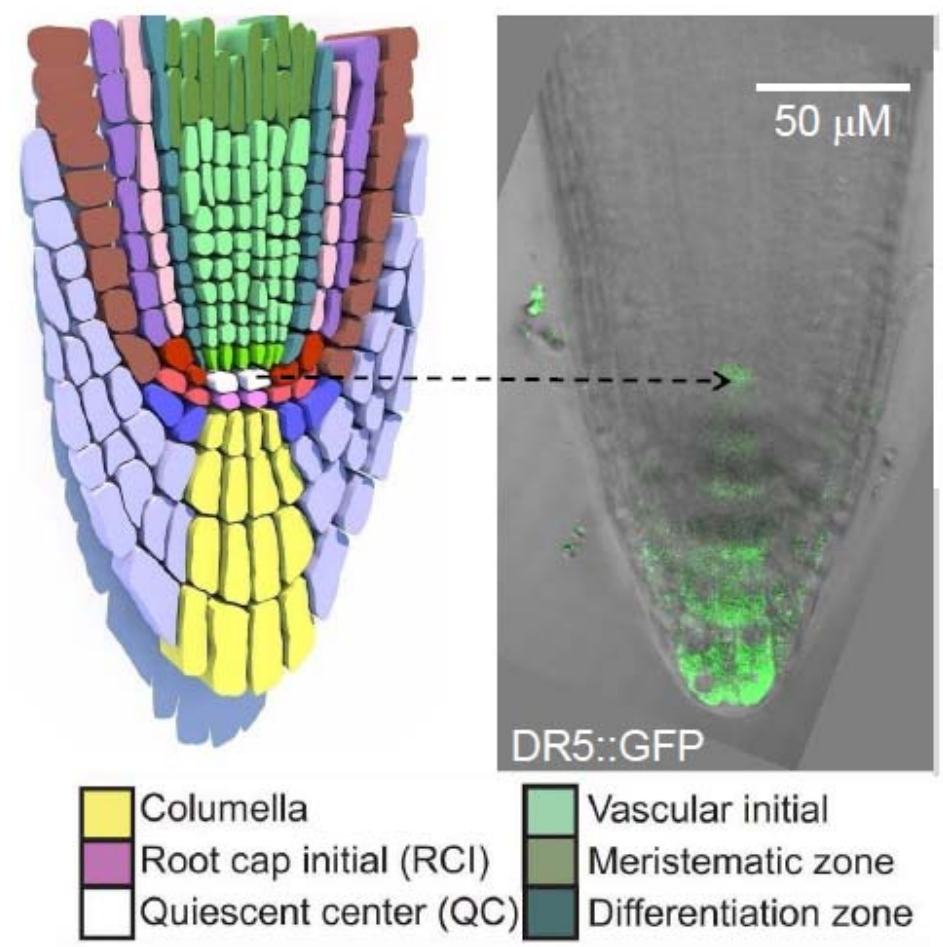

FiguRE 7. Root apical meristem structure in Arabidopsis thaliana. Different cell types are shown by different colors in the left scheme. Color code for the cell types is presented underneath. Auxin response distribution revealed by GFP fluorescence in DR5::GFP transgenic line is shown in the right confocal image. Auxin response maximum is localized in the quiescent center, high auxin response level is also observed in columella.

initials (and columella) and will simulate only the development of the root central cylinder. The initial cell configuration is shown in Figure 5, c1. The yellow cells at the upper row simulate the quiescent center. The green cells from the sides of the QC relate to cortex/endodermis initials. The green cells below the QC cells relate to vascular initials.

Alone with the geometrical structure, we also changed the rates and directions of division for different initials. The results of this analysis are shown in Figure 5, c2, c3. The most left and most right green cells in the second row have variable direction of division. The first division is in the horizontal direction (Figure 5, c2), the next division in the vertical direction (Figure 5, c3). The upper green cells divide twice slower than the cells below them.

The better arrangement of vertical cell layers in the simulating root was received with slowly dividing upper initials. In this case we observe more stable growth. Experimentally, the same pattern is observed in plant roots of different species whereas the main part of vascular cylinder consists of the cell rows taking its origin from the vascular initials. The root growth simulated from the initial data (Figure 5, c4) shows a weak instability where the structure is not exactly symmetric. The reason of unstable growth is in highly stressed QC cells. No variation in the model parameters (directions and rates of division) with the basic initial data (Fig. 5, c1) gives more stable root growth.

3.0.0.9. Growth and division of differentiated cells. In plant meristem differentiated cells can grow and divide. We begin with growth of differentiated cells without their division. The evolution of the equilibrium cell area $S_{0}$ is now described by the equation 

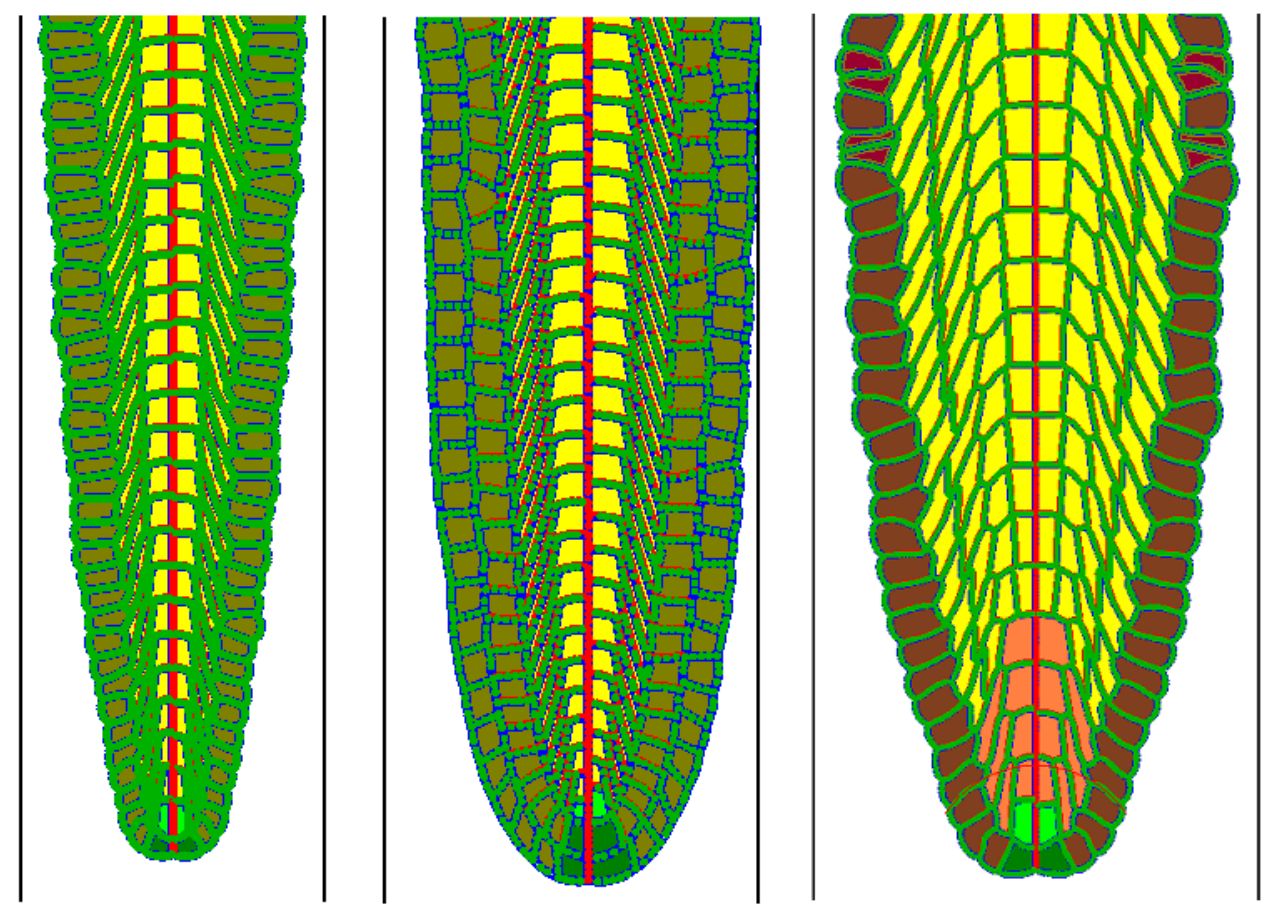

FiguRE 8. Models of root growth where differentiated cells can grow and divide. Growth of differentiated cells without division for two (left) and three (middle) layers of stem cells. Root growth with dividing differentiated cells (right). Dividing differentiated cells are rose, differentiated cells, which do not divide, are yellow and brown.

$$
\frac{d S_{0}}{d t}=\lambda e^{-a t}
$$

where $a$ is a positive constant. The growth rate is exponentially decaying in time providing limited cell growth. We still keep linear growth for stem cells $(a=0)$ with possibly a different value of $\lambda$. Examples of simulations with growing differentiated cells are shown in Figure 8 in the case of two layers of dividing cells (left) and three layers of dividing cells (middle). The shape of the root is now different in comparison with the case where differentiated cells do not grow (Section 2).

Let us now consider the case where differentiated cells can grow and divide. Their growth is described by the same formula as above. In order to determine which cells divide, we introduce the following condition. We call the tip of the root its lowest point at the axis of symmetry. When a mother cell divides, we measure the distance from the tip to the centers of mass of the daughter cells. If this distance is less than some given number $r$, then this cell will divide when its area becomes twice its initial area. This condition mimics a morphogene produced by stem cells, which determine cell division.

An example of simulations with growing and dividing differentiated cells is shown in Figure 8, right. There are four cell types: stem cells and three types of differentiated cells. Dark green stem cells in the lower row divide giving brown differentiated cells in the outer layer. The latter grow but do not divide (for this choice of parameters). Light green stem cells in the second layer divide giving rose differentiated cells above them. These cells grow and when their size become two times their initial size, they divide giving yellow differentiated cells. These cells grow but do not divide any more. Hence in the example considered here, differentiated cells divide one more time. The model (and software) allow simulations with several generations of dividing differentiated cells. 


\section{Auxin flux in the root}

Auxin regulates stem cell niche maintenance in the meristems. We use the deformable cell model described in the previous sections in order to simulate auxin distribution. Here we assume that cell growth is independent of auxin distribution. Thus, we decouple in this model root growth and auxin functioning.

We will denote by $a_{i, j}$ auxin concentration in cell $(i, j)$ (Figure 9), and similar in other cells. Along with auxin concentration, we will also consider the total auxin quantity in each cell, $A_{i, j}=a_{i, j} V_{i, j}$, where $V_{i, j}$ is the cell volume (which can change with time).

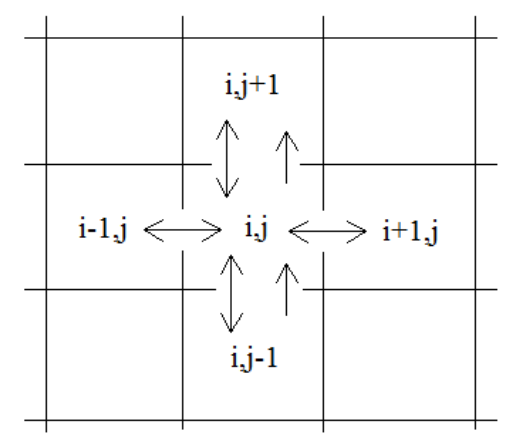

Figure 9. Schematic representation of auxin flux. Simple arrow shows polar (convective) transport, double arrow shows diffusive flux.

We consider here the reflected flow mechanism of auxin distribution in the root, which takes into account positive and negative feedbacks of auxin on its own transport. This mechanism is suggested in [20], [21] in the 1D case. Here we will test it in the 2D deformable cell model. We do not explicitly simulate PIN dynamics in this model. Each two neighboring cells exchange auxin due to diffusive flux. Auxin regulated polar transport occurs in some specific direction which should be specified. In the example shown in Figure 9, equation for $A_{i, j}$ writes:

$$
\frac{d\left(a_{i, j} V_{i, j}\right)}{d t}=d \sum_{k=i \pm 1, l=j \pm 1} S_{k, l}^{i, j}\left(a_{k, l}-a_{i, j}\right)+S_{i, j-1}^{i, j} \phi\left(a_{i, j-1}\right)-S_{i, j}^{i, j+1} \phi\left(a_{i, j}\right)-\sigma a_{i, j} V_{i, j},
$$

where $S_{k, l}^{i, j}$ is the length of the boundary between the cells $(i, j)$ and $(k, l)$,

$$
\phi(a)=\left\{\begin{array}{c}
k_{4} a, a<a_{c} \\
0, a \geq a_{c}
\end{array},\right.
$$

$d, k_{4}$ and $\sigma$ are positive constants. In the case of other cell structures, formula (4.1) remains similar with summation of diffusion flux taken with respect to all cells surrounding the cell $(i, j)$.

The first term in the right-hand sides of this equation describes auxin exchange with the surrounding cells due to usual diffusion mechanism. The fluxes are proportional to the concentration difference, summation is taken with respect to all neighboring cells. The terms, which contain the function $\phi\left(a_{i j}\right)$, describe polar auxin transport. In this example, it concerns only cells located in the central column. The value of this transport is proportional to the concentration of auxin if it is less than a critical concentration $a_{c}$ and zero otherwise. The last term in this equation describes auxin degradation. 


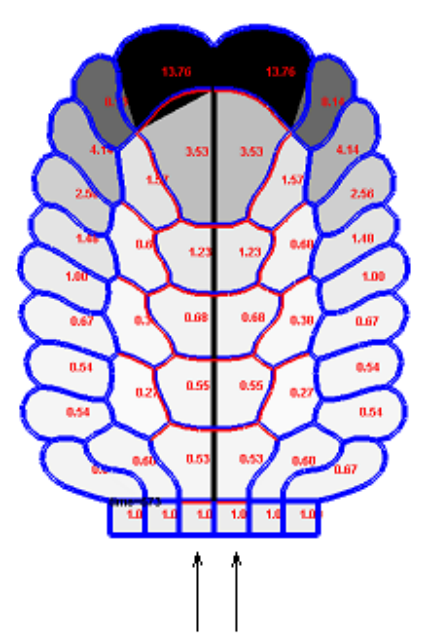

polar transport
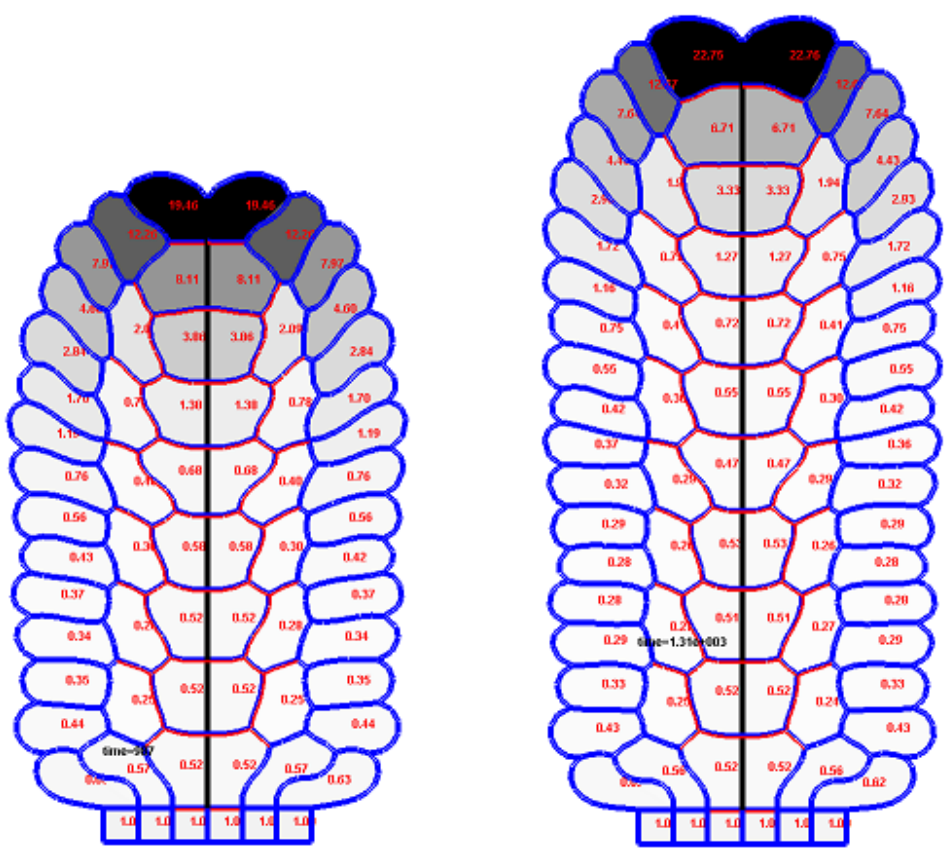

FiguRE 10. Auxin distribution in the growing root in three consecutive moments of time. The values of auxin concentrations are indicated in each cell by a number and by the level of gray. Black color corresponds to the maximal value of auxin concentration. Polar transport occurs only in the two central columns from below to above, up to the upper initial cell. The values of parameters: $d=0.1, k_{4}=0.3, a_{c}=30, \sigma=3 \cdot 10^{-5}$.

We recall that root growth in this model is independent of auxin distribution. The purpose of this modeling is to study auxin distribution during steady root growth. We now describe various patterns of auxin distribution in the process of root growth.

4.0.0.10. Steady auxin distribution with maximum at the tip. Figure 10 shows auxin distribution along the self-similar growing structure shown in Figure 5, a. We recall that there are two rows of dividing green cells, two cells in each row. This structure simulates the root meristem without collumella and the QC, so that the green cells at the top are the stems cells in the RAM. The cells in the second row have alternating directions of division, horizontal and vertical. We suppose that polar transport occurs in the vertical direction along two central rows of cells till the root end. Diffusive transport occurs between any two cells with a common boundary. Root growth in this model does not depend on auxin distribution.

Auxin enters the growing structure by the lower row of cells which have a fixed square shape. These cells serve as a support for the growing structure and also provide boundary conditions for auxin concentration which has here a constant value. Polar transport starts with the two central cells in the lower row and continues along the two central columns. Other cells in this row participate only in diffusive transport.

The auxin concentration first decreases with the distance from the base cells and then increases again with the maximum reached in the upper central cells. Its distribution has a specific fountain shape observed in [14] in a rectangular cell structure. This auxin distribution maintains in the process of root growth. The maximal auxin concentration for these values of parameters remains less than the critical value $a_{c}$, such that polar transport is not stopped by the reflected flow mechanism. The observed solution relates to the normal auxin distribution with the maximum in the stem cell niche right below the root cap. The auxin gradient can determine the length of the meristematic zone in the root meristem. 
Let us note that when a cell grows, the total auxin quantity inside it does not change (if we do not take into account auxin influx and efflux). However its concentration decreases because cell volume increases. When a cell divides, its volume is divided approximately by two, as well as total quantity of auxin. Auxin concentration, that is total quantity divided by volume, remains approximately the same in the mother cell and in the daughter cells. A small variation can take place because mechanical stresses change cell shape and volume after division.

4.0.0.11. Auxin distribution with several maxima. High auxin levels inhibits its own transport [27]. If we now decrease the critical value of auxin concentration for inhibition of polar transport, $a_{c}=3$, then it is reached at some moment of time, and the cell where it is reached stops convective flux. Figure 11 shows consecutive moments of time in root growth.

Let us analyze auxin distribution in Figure 11, left. We will denote cells in the left central column by Cell 1 (lower cell just above the square support cell), Cell 2 above it and so on till Cell 7 at the top. The concentrations of auxin in this cells are as follows:

Cell 1: 0.62, Cell 2: 1.30, Cell 3: 6.90, Cell 4: 4.83, Cell 5: 3.01, Cell 6: 1.37, Cell 7: 4.89.
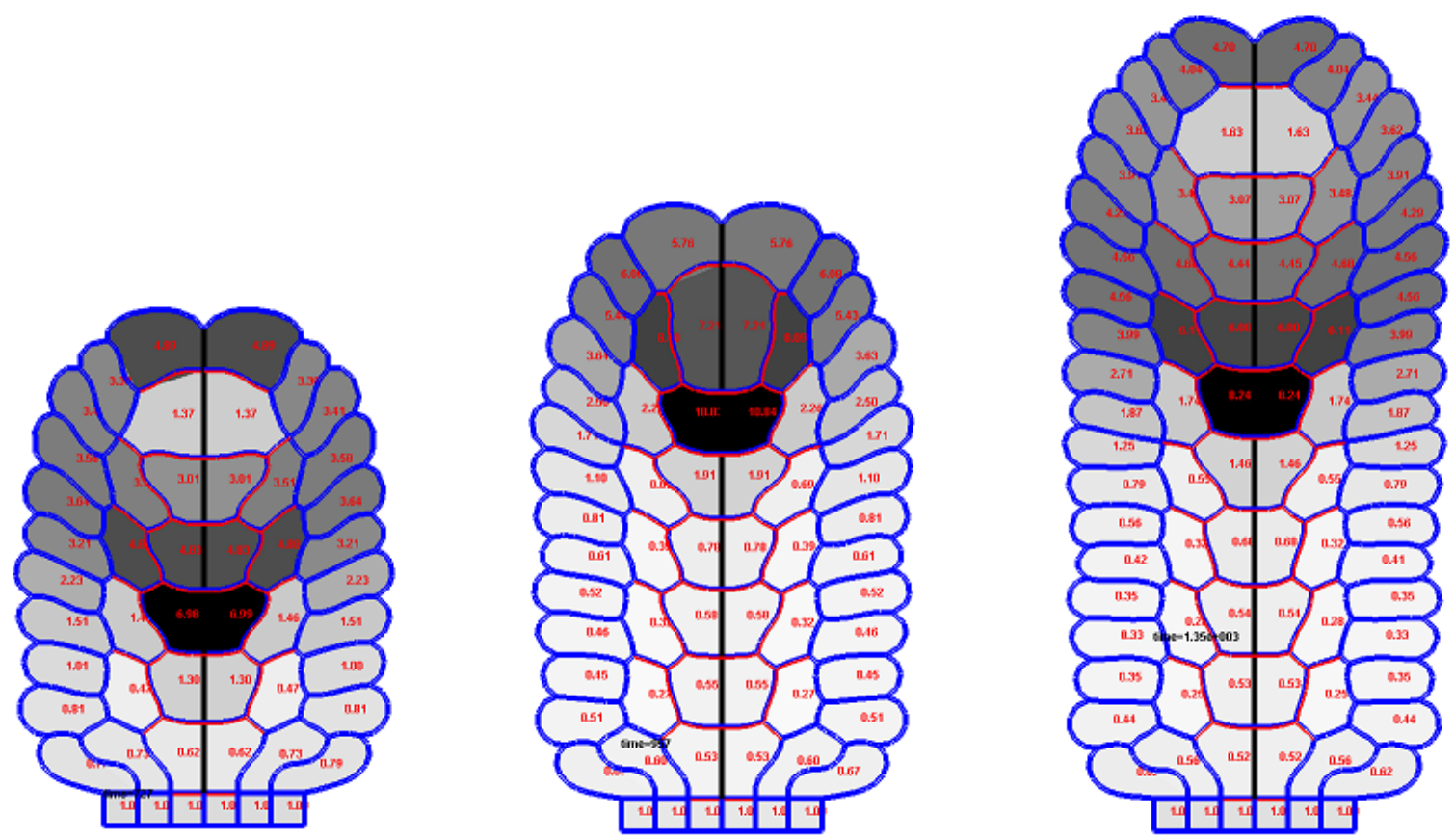

FiguRE 11. Auxin distribution in a growing root in consecutive moments of time. The pattern of cells at the tip of the root repeats periodically in time.

Cell 3 has the maximal value of auxin. Some time before, when it was the upper cell of the structure, it accumulated a high auxin concentration due to polar transport. Since the concentration in this cell is greater than the critical value $a_{c}$, polar transport here is stopped. It does not go from Cell 3 to Cell 4 . On the other hand, Cell 3 receives polar transport from Cell 2 and it also sends auxin in surrounding cells by diffusive transport. Thus the auxin level in this cell is determined by the balance between incoming polar transport and outcoming diffusive transport.

At the same time, cells continue to divide and Cell 4, then Cell 5 and the others appear above Cell 3. When a cell grows before division, increase of its area decreases auxin concentration. Cell division preserves the value of the concentration (but divides by 2 the total quantity of auxin). Hence division 
of cells above Cell 3 results in a gradual decrease in auxin concentration. Finally it become less than $a_{c}$ in Cell 6. Then polar transport from Cell 6 is again possible and auxin begins to grow into Cell 7 . As a consequence, its concentration in Cell 6 decreases, and diffusive transport from Cell 5 to Cell 6 increases. Therefore auxin concentration in Cell 5 decreases. In the same manner, we will show that diffusive transport from Cell 3 increases, auxin concentration their decreases and after some time the polar transport restarts. Auxin accumulates again in the upper cells and the whole cycle repeats (Figure 11, middle and right).

If we increase the intensity of convective transport, then several local maxima of auxin concentration are generated. As it was speculated in 1D model of reflected flow mechanism [20], the additional auxin maxima may account for lateral root primordia initiation. Periodic formation of the auxin maxima in the basal meristem of A. thaliana was shown to be correlated with the lateral root primordia development [22], [9].

\section{Discussion}

Here we present the deformable cell model for simulation of plant tissue development. Pattern formation in plants occurs in the absence of cell migration, sliding or programmed cell death. Plant development mainly coordinated by cell proliferation, expansion, and differentiation. As plant cells are closely connected to each other, coordination of cell dynamics and their mechanical properties within the tissue are very important for plant growth and development. The deformable cell model was created taking into account these features. Cell ensemble in the model is generated by dividing initials. Cell change its shape due to isotropic growth and mechanical interaction with the surrounding cells. The model solution depends on initial data, directions and rates of division. Several tools for simulation plant tissue growth has been created before. Among them 2D models of auxin transportation in the SAM [2], [15], [24], root apical meristem development [25], [26], framework for cell-based modeling of plant tissue growth and development [19]. The present deformable cell model has some features in common with the model developed in [19]: cells are represented by polygons with deformable walls, cells grow, divide and possess some intracellular concentrations. However the equations of motion, mechanical properties of the walls, the equations for concentrations and some other details are different ${ }^{1}$.

Using the deformable cell model we investigated formation of self-similar cell structures. A growing cell structure $S(t)$ is self-similar if for any time t it can be obtained from $S(t-\tau)$ by translation in space. By numerical simulations we showed that self-similar cell structures exist under specific and rather restrictive conditions on cell organization, division angles and mechanical properties. Repetitive pattern is often observed in nature. Plant modules (shoots, roots) are the self-similar structures, as they are repeated in the plant life cycle. Their origins, plant meristems represent self-similar cell structures which reproduce the same cell organization during their functioning. We investigated here formation of self-similar cell structures simulating functioning of shoot and root meristems.

Cell dynamics in the SAM was simulated from the initial cellular layout (Figure 6) containing three layers of initials. The directions of division were chosen for each layers in such a way that the self-similar cell structure emerges in the model solution. In numerical simulation we observed formation of continuous L1 and L2 layers as well as highly organized L3. In real SAM, L3 cells divide in different directions providing volume growth [28]. This dynamics can be simulated in the elastic cell model, however the solution may not be self-similar.

Despite rather simple structure of the root apical meristem, cell dynamics their is quite complex with several types of initials characterized by different directions and rates of division. To simulate some of the RAM-specific features we created several deformable cell model variants. First, in the model without root cap initial we simulated development of vascular cylinder. In this model, we also investigate maintenance of the QC cells. Analysis of the model allows us to make some hypothesis on the rules of cell divisions in

\footnotetext{
${ }^{1}$ The authors of this paper were not aware of the work [19] during the software development.
} 
the RAM. Among them the relative rates and directions of divisions for different initials and mechanical properties of cell walls.

Quiescent center in the RAM is covered by the root cap which preserve them from exterior medium. In the real RAM the descendants of the root cap initials are sloughed away in the course of root growth. To simulate root cap growth we created another RAM model variant with possibility to remove the outer columella layer. This is the first model simulating such kind of dynamics in RAM. In numerical simulation we observed formation of stretched layers of the columella. These results are not presented in this work and will be published elsewhere. Additional rules of cell dynamics in the root cap should be introduced in order to simulate the real RAM dynamics.

The deformable RAM model was tested to simulate auxin distribution in the root. We simulated auxin transport under the mechanism of the reflected flow [20], which considers positive and negative regulation of auxin transport by auxin itself. We simplified the mechanism presented in [20] taking into account only negative regulation described here as the threshold function (4.1). In the self-similar model solutions auxin maxima and gradients are generated which may influence cell dynamics in the RAM. Here we show how additional auxin maxima can provide root branching. It has been shown previously [22], [9] that auxin maxima in the basal meristem predetermine initiation of lateral root. In [20] it has been proposed that these maxima emerge under the mechanism of reflected flow. Here we test this hypothesis in the 2D elastic cell model. These simulations show that the deformable cell model can be a powerful tool for simulating developmental control of plant tissue growth.

Acknowledgements. The work was supported by the research grant ANR Bimod, French-Russian project PICS, Mathematical Modeling of Blood Diseases, and grant no. 14.740.11.0877 of the Ministry of Education and Research of the Russian Federation, Investigation of Spatial and Temporal Structures in Fluids with Applications to Mathematical Biology. MVV was supported by RFBR grants 11-04-01254a, 12-04-33112-mol-a-ved, Dynasty Foundation grant for young biologists, SBRAS project 80, SS-5278.2012.4.

\section{References}

[1] L.R. Band, D.M. Wells, A. Larrieu, J. Sun, A.M. Middleton. Root gravitropism is regulated by a transient lateral auxin gradient controlled by a tipping-point mechanism. PNAS, 109 (2012), no. 12, 4668-4473.

[2] E.M. Bayer, R.S. Smith, T. Mandel, N. Nakayama, M. Sauer, P. Prusinkiewicz, C. Kuhlemeier. Integration of transportbased models for phyllotaxis and midvein formation. Genes Dev., 23 (2009), no. 3, 373-384.

[3] N. Bessonov, V. Volpert. Dynamic models of plant growth. Publibook, Paris, 2006.

[4] N. Bessonov, N. Morozova, V. Volpert. Modelling of branching patterns in plants. Bull. Math. Biology, 70 (2008), no. 3, 868-893.

[5] G.D. Bilsborough, A. Runions, M. Barkoulas, H.W. Jenkins, A. Hasson, C. Galinha, P. Laufs, A. Hay, P. Prusinkiewicz, M. Tsiantis. Model for the regulation of Arabidopsis thaliana leaf margin development. PNAS, 108 (2011), no. 8, 34243429 .

[6] I. Blilou, J. Xu, M. Wildwater, V. Willemsen, I. Paponov, J. Friml, R. Heidstra, M. Aida, K. Palme, B. Scheres. The PIN auxin efflux facilitator network controls growth and patterning in Arabidopsis roots. Nature, 433(7021) (2005), 39-44.

[7] V. Brukhin, N. Morozova. Plant growth and development - basic knowledge and current views. Math. Model. Nat. Phenom., 6 (2011), no. 2, 1-53.

[8] G. Brunoud, D.M. Wells, M. Oliva, A. Larrieu, V. Mirabet, A.H. Burrow, T. Beeckman, S. Kepinski, J. Traas, M.J. Bennett, T. Vernoux. A novel sensor to map auxin response and distribution at high spatio-temporal resolution. Nature, 482(7383) (2012), 103-106.

[9] I. De Smet, T. Tetsumura, B. De Rybel, N.F. Frey, L. Laplaze, I. Casimiro, R. Swarup, M. Naudts, S. Vanneste, D. Audenaert, D. Inze, M.J. Bennett, T. Beeckman. Auxin-dependent regulation of lateral root positioning in the basal meristem of Arabidopsis. Development, 134 (2007), no. 4, 681-690.

[10] L. Dolan, K. Janmaat, V. Willemsen, P. Linstead, S. Poethig, K. Roberts, B. Scheres. Cellular organisation of the Arabidopsis thaliana root. Development, 119 (1993), 71-84.

[11] L. Forest, J. Demongeot. Cellular modelling of secondary radial growth in conifer trees: application to Pinus radiata (D Don). Bull. Math. Biol. 68 (2006), 753-784.

[12] M.G. Heisler, C. Ohno, P. Das, P. Sieber, G.V. Reddy, J.A. Long, E.M. Meyerowitz. Patterns of auxin transport and gene expression during primordium development revealed by live imaging of the Arabidopsis inflorescence meristem. Curr Biol., 2005. 15(21), 1899-1911.

[13] K. Jiang, L.J. Feldman. Regulation of root apical meristem development. Ann. Rev. Cell Dev. Biol., 21 (2005), $485-509$. 
[14] V.A. Grieneisen, J. Xu, A.F. Marle, P. Hogeweg, B. Scheres. Auxin transport is sufficient to generate a maximum and gradient guiding root growth. Nature 2007, 449(7165), 1008-1013.

[15] H. Jonsson, M.G. Heisler, B.E. Shapiro, E.M. Meyerowitz, E. Mjolsness. An auxin-driven polarized transport model for phyllotaxis. PNAS, 103 (2006), no. 5, 1633-1638.

[16] E.M. Kramer. A mathematical model of pattern formation in the vascular cambium of trees. J. Theor. Biol. (2002) $216,147-158$.

[17] P. Krupinski, H. Jonsson. Modeling auxin-regulated development. Cold Spring Harb Perspect Biol., 2010 Feb. 2(2): a001560.

[18] M. Lucas, L. Laplaze, M.J. Bennett. Plant systems biology: network matters. Plant Cell Environ., 34 (2011), no. 4, $535-353$.

[19] R.M. Merks, M. Guravage, D. Inze, G.T. Beemster. VirtualLeaf: an open-source framework for cell-based modeling of plant tissue growth and development. Plant Physiol., 155 (2011), no. 2, 656-666.

[20] V.V. Mironova, N.A. Omelyanchuk, E.S. Novoselova, A.V. Doroshkov, F.V. Kazantsev, A.V. Kochetov, N.A. Kolchanov, E. Mjolsness, V.A. Likhoshvai. Combined in silico/in vivo analysis of mechanisms providing for root apical meristem self-organization and maintenance. Ann. Bot., 110 (2012), no. 2, 349-360.

[21] V.V. Mironova, N.A. Omelyanchuk, G. Yosiphon, S.I. Fadeev, N.A. Kolchanov, E. Mjolsness, V.A. Likhoshvai. A plausible mechanism for auxin patterning along the developing root. BMC Syst. Biol., 2010 Jul 21;4:98.

[22] M.A. Moreno-Risueno, J.M. Van Norman, A. Moreno, J. Zhang, S.E. Ahnert, P.N. Benfey. Oscillating gene expression determines competence for periodic Arabidopsis root branching. Science, 329 (2010) no. 5997, 1306-1311.

[23] M. Sauer, J. Balla, C. Luschnig, J. Wisniewska, V. Reinohl, J. Friml, E. Benkova. Canalization of auxin flow by Aux/IAA-ARF-dependent feedback regulation of PIN polarity. Genes \& Dev., 20 (2006), 2902-2911.

[24] P. Sahlin, B. Soderberg, H. Jonsson. Regulated transport as a mechanism for pattern generation: capabilities for phyllotaxis and beyond. J. Theor. Biol., 258 (2009), no. 1, 60-70.

[25] J. Szymanowska-Pulka, I. Potocka, J. Karczewski, K. Jiang, J. Nakielski, L.J. Feldman. Principal growth directions in development of the lateral root in Arabidopsis thaliana. Ann. Bot., 110 (2012), no. 2, 491-501.

[26] J. Szymanowska-Pulka. Application of a changing field of growth rates to a description of root apex formation. J. Theor. Biol., 247 (2007), no. 4, 650-656.

[27] A. Vieten, S. Vanneste, J. Wisniewska, E. Benkova, R. Benjamins, T. Beeckman, C. Luschnig, J. Friml. Functional redundancy of PIN proteins is accompanied by auxin dependent cross-regulation of PIN expression. Development, 132 (2005), no. 20, 4521-4531.

[28] L. Williams, J.C. Fletcher. Stem cell regulation in the Arabidopsis shoot apical meristem. Curr. Opin. Plant Biol., 8 (2005), no. 6, 582-586. 